\title{
Estudo comparativo entre os níveis de glicemia venosa e glicemia capilar
}

\author{
Bárbara de Castro Borges ${ }^{1}$ \\ Tania Cristina Andrade ${ }^{2}$
}

\section{Resumo}

A manutenção da glicemia normal depende principalmente da capacidade funcional das células pancreáticas em secretar insulina e da sensibilidade tecidual à ação da insulina. O objetivo deste trabalho é realizar um estudo de comparação entre os níveis de glicemia venosa e de glicemia capilar de um mesmo indivíduo para verificar se elas são compatíveis. Foi avaliada a eficácia da monitorização glicêmica capilar (realizada por glicosímetro) comparada à dosagem glicêmica venosa. Na amostra total, não houve diferença significativa entre a glicemia capilar e a venosa, quando realizadas em jejum e simultaneamente. Pode-se concluir que o uso da medida de glicemia capilar deve ser fortemente estimulado na prática clínica para a monitorização domiciliar do paciente, uma vez que o controle rigoroso dos níveis de glicemia contribui para a redução das principais complicações do diabetes mellitus. O uso do glicosímetro tem impacto benéfico na redução dos riscos e complicações em pacientes diabéticos.

Palavras-chave: Glicemia capilar. Glicemia venosa. Glicosímetro. Diabetes mellitus.

\section{Introdução}

Diabetes mellitus é um grupo de desordens metabólicas que apresenta como característica comum à hiperglicemia, ocorrendo devido a defeitos na secreção da insulina, na sua ação ou nos dois mecanismos (KUMAR et al., 2005). A hiperglicemia crônica causa dano, disfunção e falência de vários órgãos (GROSS et al., 2002).

Diabetes mellitus tipo 1 caracteriza-se pela destruição das células beta pancreáticas (células de Langerhans); é um distúrbio catabólico, com ausência de insulina,

1 Biomédica. Graduada pelo UniCEUB. E-mail: babicastro@gmail.com

2 Bióloga. Especialista em Análises Clínicas. Mestre em Biologia Molecular. Professora do UniCEUB, FACES. E-mail: taniaandrade21@yahoo.com.br 
elevação da glicemia, degradação de lipídios e proteínas (MUNDIM et al., 2004). Os pacientes passam a depender de insulina para a sobrevivência (DAMIANI, 2000).

Diabetes mellitus tipo 2 é uma doença crônica (FERRAZ; MAIA; ARAUJO, 2004); está associada à presença de obesidade e resulta da combinação de defeito secretório da célula beta e falha na ação periférica da insulina (GELONEZE; LAMOUNIER; COELHO, 2006).

A glicemia é o nível de glicose que está presente no sangue, mantido geralmente entre 70 e 120 mg/dL (KUMAR et al., 2005). A manutenção da glicemia normal depende da capacidade das células pancreáticas em secretar insulina e da sensibilidade tecidual à ação da insulina (VASQUES et al., 2008).

O rastreamento de diabetes deve ser realizado em todo indivíduo com mais de 45 anos de idade a cada 3 anos. Os diagnósticos baseiam-se na glicose plasmática de jejum de $2 \mathrm{~h}$ após sobrecarga oral de $75 \mathrm{~g}$ de glicose e na medida da glicose plasmática casual. A dosagem de glicemia plasmática de jejum é mais econômica e de fácil execução e apresenta um menor coeficiente de variação do que o Teste Oral de Tolerância à Glicose (TOTG).

O TOTG deve ser empregado como método diagnóstico de diabetes sempre que possível, especialmente quando os valores de glicose plasmática em jejum estiverem entre $110 \mathrm{mg} / \mathrm{dL}$ e $126 \mathrm{mg} / \mathrm{dL}$; em indivíduos com mais de 65 anos, independente dos valores de glicose plasmática; e em gestantes (GROSS et al., 2002).

Os níveis da glicemia sofrem influência de vários fatores, e estão sujeitos à contínua flutuação ao longo do dia (INSTITUTO DE ANÁLISES CLÍNICAS DE SANTOS, 2007). Podem ser medidos por meio de exames realizados em laboratório ou de exame realizado em um glicosímetro (VERGANI; GEBRIM; COSTA, 2004).

Após o diagnóstico de diabetes, os pacientes iniciam diversos tipos de tratamento. A medida da hemoglobina glicada é a escolha para controle em longo prazo; reflete o controle glicêmico de 2 a 3 meses anteriores e sua percentagem depende dos níveis de glicose no sangue, da duração da exposição da hemoglobina à glicose e ao tempo de vida dos eritrócitos (GROSS et al., 2002).

A avaliação da glicemia ao longo do dia é uma estratégia importante para se obter o melhor controle metabólico possível (GROSS et al., 2002). A automonito- 
rização da glicose é indicada para todo o paciente tratado com insulina ou agentes anti-hiperglicemiantes orais (AMERICAN DIABETES ASSOCIATION, 2001). Os pacientes devem receber treinamento ambulatorial para o correto manuseio do equipamento, pois vários fatores podem alterar os resultados obtidos nesses aparelhos, como o volume da amostra de sangue e o manuseio incorreto da fita reagente e do glicosímetro (MIRA; CANDIDO; YALE, 2006).

O objetivo deste trabalho foi realizar um estudo comparativo entre os níveis de glicose venosa e capilar de um mesmo indivíduo, verificar se há diferença estatisticamente significante entre as dosagens e testar a eficácia dos glicosímetros usados por pessoas diabéticas como forma de controle da glicemia.

\section{Metodologia}

Este estudo foi submetido e homologado pelo Comitê de Ética em Pesquisa do UniCEUB. Foram coletadas amostras de 35 pacientes voluntários escolhidos ao acaso, sendo $4 \mathrm{~mL}$ de sangue venoso e uma amostra de sangue capilar por uso de lancetas. A amostra venosa foi colocada em tubos de coleta secos - os tubos foram centrifugados durante 10 minutos a 10.000 rpm para separação do soro- utilizados para a realização da dosagem de glicemia pela metodologia enzimática - Kit DOLES $^{\circledast}$, que já fornece os padrões e os reagentes enzimáticos.

A coleta de sangue com ajuda de lancetas foi realizada em ponta de dedo. Uma gota de sangue foi colocada em fita específica para dosagem de glicose capilar em glicosímetros. O aparelho (Accu-Check ${ }^{\circledast}$ ) fornece o resultado da glicemia em 15 segundos. Para a verificação de diferenças significativas entre os grupos, foi utilizado o teste $T$ student e, para a confecção dos gráficos, foi utilizado o programa GRAFPAD Prisma (versão 5.0).

\section{Resultados}

Todos os voluntários avaliados foram informados dos objetivos desta pesquisa e assinaram o Termo de Consentimento Livre e Esclarecido aprovado pelo Comitê de Ética em Pesquisa do UniCEUB. 
$\mathrm{Na}$ avaliação da eficácia da monitorização glicêmica capilar, quando comparada à venosa, alguns pacientes apresentaram valores de glicemia capilar superior à venosa (Tabela 1), enquanto outros apresentaram a glicemia venosa com valores superiores à capilar (Tabela 2). Dos 35 pacientes incluídos neste estudo, apenas 2 não apresentaram variação entre as duas glicemias medidas.

Foi calculada uma média e um desvio padrão a partir da diferença percentual encontrada através da comparação dos valores glicêmicos venosos e capilares.

Tabela 1 - Variação glicêmica de pacientes com glicemia capilar superior à glicemia venosa $(n=19)$.

\begin{tabular}{|c|c|}
\hline Dados Estatísticos & Diminuição da venosa em relação à capilar (\%) \\
\hline Variação percentual & $1,0-49,0$ \\
\hline Média & 21,7 \\
\hline Desvio padrão & 14,9 \\
\hline
\end{tabular}

Tabela 2 - Variação glicêmica de pacientes com glicemia venosa superior à glicemia capilar $(n=14)$.

\begin{tabular}{|c|c|}
\hline Dados Estatísticos & Diminuição da capilar em relação à venosa (\%) \\
\hline Variação percentual & $2,5-24,6$ \\
\hline Média & 16,0 \\
\hline Desvio padrão & 7,2 \\
\hline
\end{tabular}

A análise estatística desses dados mostrou diferenças significativas entre os grupos avaliados, conforme mostram os gráficos 1 e 2 . 
Gráfico 1 - Variação da dosagem glicêmica com diminuição da glicemia venosa em relação à glicemia capilar. ${ }^{\star} \mathrm{p}<0,05$ sendo $\mathrm{p}<0,0001$ em relação à glicemia capilar.

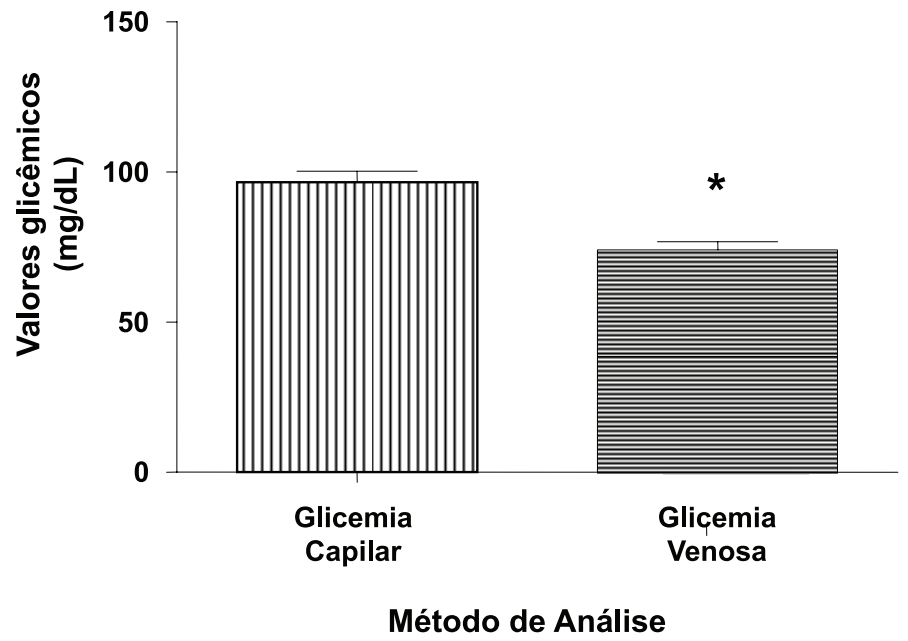

Gráfico 2 - Variação da dosagem glicêmica com aumento da glicemia venosa em relação à glicemia capilar. ${ }^{*} \mathrm{p}<0,05$ sendo $\mathrm{p}=0,0099$ em relação à glicemia capilar.

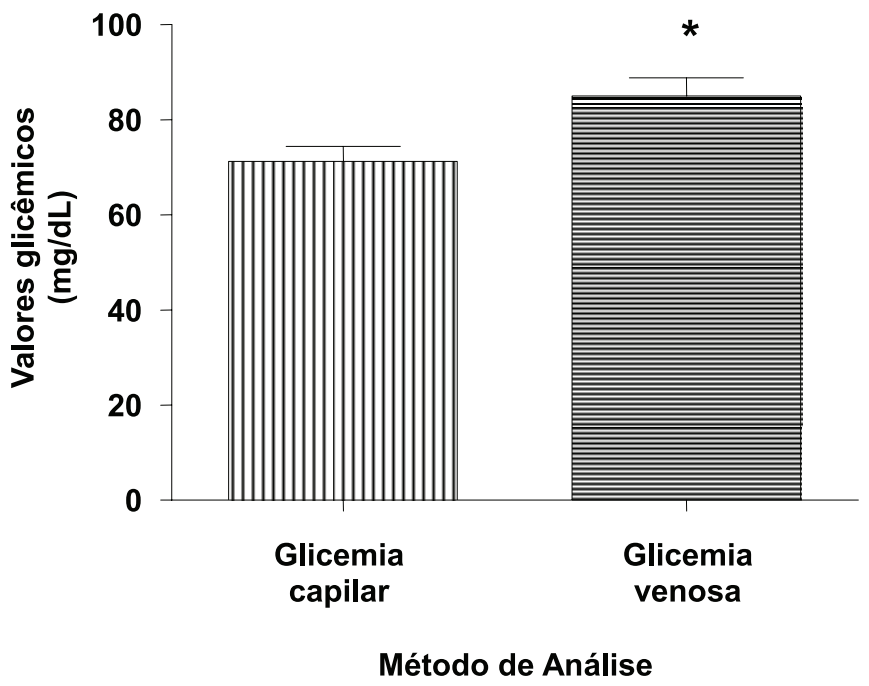


Após a análise em separado, todos os 33 pacientes foram incluídos em um único grupo para comparação entre a média de todas as glicemias. Essa análise não mostrou diferença significativa entre as dosagens, apesar da glicemia capilar mostrar, em média, valores maiores que os encontrados na dosagem venosa (Gráfico 3).

Gráfico 3 - Variação da dosagem glicêmica, considerando todos os pacientes.

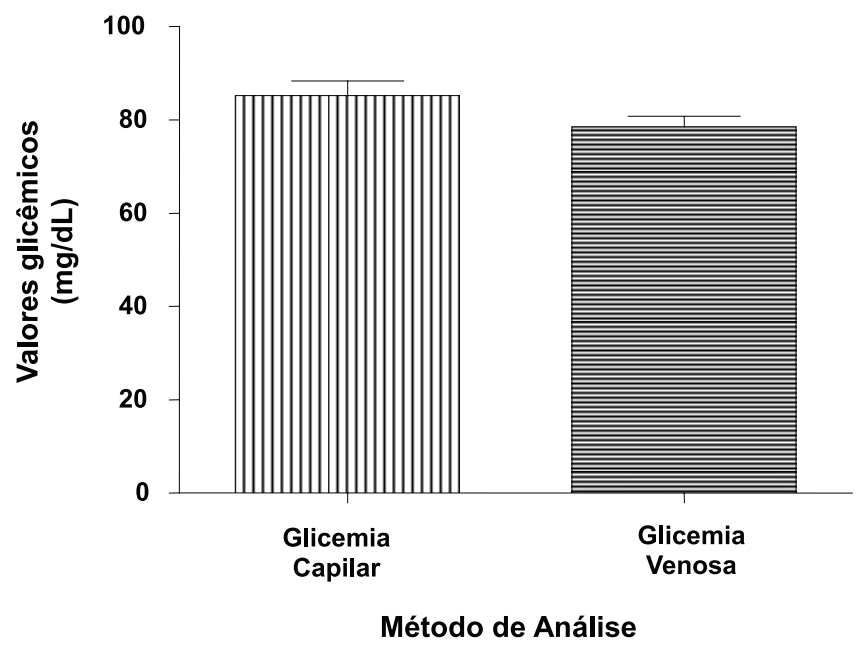

\section{Discussão}

Pacientes diabéticos utilizam glicosímetros para fazer o controle da variação dos níveis de glicose durante o dia; normalmente esses aparelhos são bastante confiáveis. Porém podem ocorrer erros devido à alteração nas fitas reagentes, quantidade de sangue disponível e aparelho descalibrado.

Analisando os dados brutos dos 33 pacientes, não houve diferença significativa entre as glicemias quando estas foram realizadas em jejum e simultaneamente sendo condizente com o estudo realizado por Pereira (2006) e seus colaboradores.

Já a análise dos dados separados mostrou que as variações encontradas são significativas; esse fato parece levantar dúvidas sobre a confiabilidade dos glicosímetros em refletir a glicemia do paciente. Essa diferença, para o grupo com valores 
capilares inferiores, pode indicar que o paciente não precisa de uma medicação imediata; já para o grupo com variação contrária, pode levar o paciente a uma medicação indevida e, talvez, a uma hipoglicemia.

Para estudar melhor esse tipo de problema, precisaríamos de uma análise adicional a este trabalho, na qual um mesmo paciente fosse avaliado em dias diferentes, quanto à variação entre glicemia capilar e venosa. Isso poderia mostrar se tal variação está ligada ao metabolismo individual ou ao uso dos aparelhos glicosímetros.

\section{Conclusão}

Com base nos resultados obtidos, pode-se concluir que o uso da medida de glicemia capilar deve ser estimulado para a monitorização domiciliar do paciente.

A análise geral deste trabalho não mostrou diferença significativa entre as glicemias avaliadas com todos os 33 pacientes no mesmo grupo, o que nos leva a crer que o uso do aparelho glicosímetro é benéfico na redução de complicações em pacientes diabéticos.

Os pacientes que utilizam esse tipo de aparelho devem ser devidamente orientados quanto ao seu manuseio correto, já que este teste é essencial para o controle dos níveis glicêmicos e para uma vida mais saudável.

\section{Comparative study between venous glycemia and capillary glycemia}

\section{Abstract}

The maintenance of normal blood glucose mainly depends on the functional capacity of pancreas cells in secreting insulin and tissue sensitivity to the insulin action. The objective of this work is a study of comparison between the levels of venous glycemia and capillary glycemia of the same individual. It was evaluated the effectiveness of monitoring capillary glycemia (by a glucometer) compared to intravenous glycemia The total sample showed there was no significant difference between the venous and capillary blood glycemia, when carried out in fasting and 
simultaneously. It was concluded that the use of capillary blood glucose measure should be strongly encouraged in clinical practice for monitoring the patient's home, since the strict control of glucose levels contributes to reducing the major complications of diabetes mellitus. The use of the apparatus glucometer has beneficial impact on reducing the risks arising from complications in diabetic patients.

Keywords: Capillary glycemia. Venous glycemia. Glucometer. Diabetes mellitus.

\section{Referências}

AMERICAN DIABETES ASSOCIATION. Standards of medical care for patients with diabetes mellitus: position statement. Diabetes Care, Washington, n. 24. p. 33-43, 2001. Suplemento

DAMIANI, D. Critérios diagnósticos no diabetes mellitus. Revista da Associação Médica Brasileira, São Paulo, v. 46, n. 4, out./dez. 2000.

FERRAZ, D. P.; MAIA, F. F. R.; ARAUJO, L. R. Glicemia capilar em ponta do dedo versus lóbulo de orelha: estudo comparativo dos valores resultantes e preferências dos pacientes. Arquivos Brasileiros de Endocrinologia e Metabologia, São Paulo, v. 48, n. 3, 2004.

GELONEZE, B.; LAMOUNIER, R. N.; COELHO, O. R. Hiperglicemia pósprandial: tratamento do seu potencial aterogênico. Arquivos Brasileiros de Cardiologia, São Paulo, v. 87, n. 5, 2006.

GROSS, J. L. et al. Diabetes Melito: diagnóstico, classificação e avaliação do controle glicêmico. Arquivos Brasileiros de Endocrinologia e Metabologia, São Paulo, v. 46, n. 1, 2002.

INSTITUTO DE ANÁLISES CLÍNICAS DE SANTOS. Diabetes e sua saúde. Disponível em: <http://www.iacs.com.br/informacoesaopaciente_ diabetes. asp.htm>. Acesso em: 07 nov. 2007.

KUMAR, V. et al. Patologia: bases patológicas das doenças. Rio de Janeiro: Elsevier, 2005.

MUNDIM, D. F. et al. Fisiopatologia. 6. ed. Rio de Janeiro: Guanabara Koogan, 2004. 
PEREIRA, G.R. et al. Análise comparativa dos níveis de glicose capilar x glicose venosa. Revista Newslab, São Paulo, n. 79, 2006.

VASQUES, A. C. J. et al. Análise crítica do uso dos índices do Homeostasis Model Assessment (HOMA) na avaliação da resistência à insulina e capacidade funcional das células-b pancreáticas. Arquivos Brasileiros de Endocrinologia e Metabologia, São Paulo, v. 52, n. 1, 2008.

VERGANI, M. I. C.; GEBRIM, A.; COSTA, P. C. A. Diabetes: nós cuidamos. 2004. Disponível em: <http://www.diabetesnoscuidamos.com.br/ conteudo. asp?id=58.htm $>$. Acesso em: 07 nov. 2008. 
Para publicar na revista Universitas Ciências da Saúde, entre no endereço eletrônico www.publicacoesacademicas.uniceub.br. Observe as normas de publicação, facilitando e agilizando o trabalho de edição. 